\title{
Structural relaxation in quantum supercooled liquids: A mode-coupling approach
}

Cite as: J. Chem. Phys. 154, 014502 (2021); https://doi.org/10.1063/5.0032085

Submitted: 06 October 2020 . Accepted: 13 December 2020 . Published Online: 05 January 2021

Ankita Das, (D) Eran Rabani, (D) Kunimasa Miyazaki, and (D) Upendra Harbola

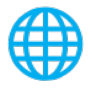

\section{ARTICLES YOU MAY BE INTERESTED IN}

Physics of phonon-polaritons in amorphous materials

The Journal of Chemical Physics 154, 014501 (2021); https://doi.org/10.1063/5.0033371

Liquid-liquid transition and polyamorphism

The Journal of Chemical Physics 153, 130901 (2020); https://doi.org/10.1063/5.0021045

Dynamical phase transitions and their relation to structural and thermodynamic aspects of glass physics

The Journal of Chemical Physics 153, 090901 (2020); https://doi.org/10.1063/5.0006998

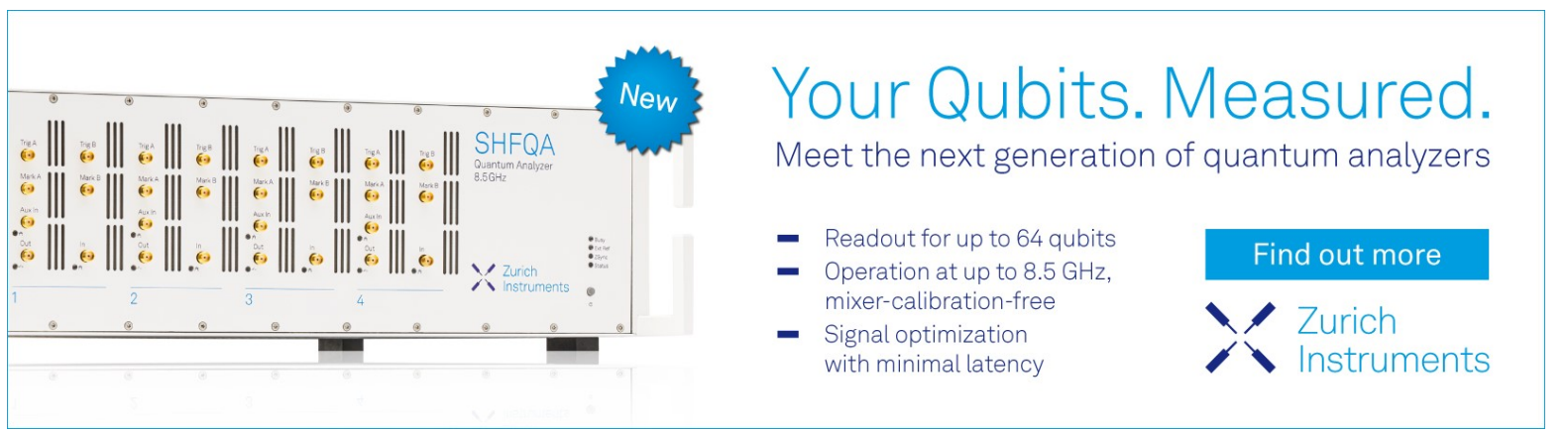




\title{
Structural relaxation in quantum supercooled liquids: A mode-coupling approach
}

\author{
Cite as: J. Chem. Phys. 154, 014502 (2021); doi: 10.1063/5.0032085 \\ Submitted: 6 October 2020 - Accepted: 13 December 2020 • \\ Published Online: 5 January 2021
}

\begin{abstract}
Ankita Das, ${ }^{1}$ (D) Eran Rabani, ${ }^{2}$ (D) Kunimasa Miyazaki, ${ }^{3}$ (D) and Upendra Harbola ${ }^{1, a)}$ (D)
AFFILIATIONS

${ }^{1}$ Inorganic and Physical Chemistry, Indian Institute of Science, Bangalore 560012, India

${ }^{2}$ Department of Chemistry, University of California, and Materials Sciences Division, Lawrence Berkeley National Laboratory, Berkeley, California 94720, USA and The Sackler Center for Computational Molecular and Materials Science, Tel Aviv University, Tel Aviv 69978, Israel

${ }^{3}$ Department of Physics, Nagoya University, Nagoya 464-8602, Japan
\end{abstract}

a) Author to whom correspondence should be addressed: uharbola@iisc.ac.in

\begin{abstract}
We study supercooled dynamics in a quantum hard-sphere liquid using quantum mode-coupling formulation. In the moderate quantum regime, classical cage effects lead to slower dynamics compared to the strongly quantum regime, where tunneling overcomes classical caging, leading to faster relaxation. As a result, the glass transition critical density can become significantly higher than for the classical liquids. A perturbative approach is used to solve time dependent quantum mode-coupling equations to study in detail the dynamics of the supercooled liquid in the moderate quantum regime. Similar to the classical case, the relaxation time shows the power-law increase with the increase in the density in the supercooled regime. However, the power-law exponent is found to be dependent on the quantumness; it increases linearly as the quantumness is increased in the moderate quantum regime.
\end{abstract}

Published under license by AIP Publishing. https://doi.org/10.1063/5.0032085

\section{INTRODUCTION}

Many liquids undergo glass transition when supercooled below its freezing temperature, bypassing the formation of a crystalline state. The time scale for structural relaxation in the supercooled liquid increases very rapidly on cooling. ${ }^{1}$ Thus, supercooled liquids are characterized by high shear viscosity. Experimentally, the glass transition is assumed to take place when viscosity is $O\left(10^{13} \mathrm{P}\right)$.

Mode-coupling theory (MCT) is a generalized hydrodynamic approach to study supercooled dynamics in simple liquids. ${ }^{3}$ MCT deals with structural dynamics of liquids encoded in the time evolution of density correlation functions. ${ }^{4-7}$ The important result of MCT consists of a self-consistent expression for generalized transport coefficients in terms of slowly decaying density correlations. The theory has been applied successfully to study liquid-glass transition in one- and two-component liquids. ${ }^{8}$ The relation between structural relaxation in supercooled liquids and frequency-dependent specific heat has been studied ${ }^{12}$ using MCT.
Most of the work on glass transition has focused on the classical regime of liquid state behavior, where the de Broglie thermal wavelength associated with a particle is significantly smaller than the particle size. As most of the glass forming liquids fall in the classical regime, it is clear that classical approximation is generally justified. However, there are several important and interesting examples, such as superfluid helium under high pressure, where quantum fluctuation and glassiness coexist. There are clear deviations from classical predictions; for example, temperature dependence of specific heat at a low temperature $(1 \mathrm{~K}-10 \mathrm{~K})$ that has been attributed to quantum tunneling. ${ }^{13-15}$ In any case, at low temperatures, one expects quantum features to show up.

To explore quantum effects on supercooled dynamics, MCT has been extended to the quantum regime known as quantum modecoupling theory (QMCT). ${ }^{16,17}$ As intuition may suggest that large quantum fluctuations serve to inhibit glass formation as tunneling and zero-point energy allow particles to traverse barriers and facilitate movement, interestingly, however, both ring-polymer molecular dynamics (RPMD) and QMCT indicate that the dynamical phase 
diagram of glassy quantum fluids is reentrant; low quantum fluctuations promote the glass transition and a further increase in quantum fluctuations leads to the inhibition to glass formation. ${ }^{18}$

In the present work, our goal is to study quantum effects on the dynamics in a supercooled state of a quantum liquid. We present a detailed analysis of the dynamics in the moderate quantum regime where the quantum effects assist in the formation of the glass phase, as opposed to the strong quantum regime where quantum fluctuations tend to avoid the glass phase. ${ }^{18}$ In Sec. II, we describe essential features of quantum dynamics of density-correlation near liquid-glass transition using QMCT. We discuss our results in Sec. III and conclude the work in Sec. IV.

\section{BASIC EQUATION OF QMCT}

QMCT is a hydrodynamic theory ${ }^{17,19}$ that is based on the dynamics of hydrodynamic variables $\{\rho$, g $\}$, where $\rho$ is the mass density and $g$ is the momentum density. The dynamics of the density fluctuations in supercooled liquids and the subsequent structural arrest provide an idea of the glass transition.

The prime goal of the theory is to describe time evolution of quantum density correlation defined as

$$
C_{\rho \rho}(q, t)=\frac{1}{N}\langle\hat{\rho}(-q, 0) \hat{\rho}(q, t)\rangle,
$$

where $\langle\cdots\rangle$ denotes quantum mechanical ensemble average and the density operator is $\hat{\rho}(q)=\sum_{i=1}^{N} e^{i q \cdot \hat{r}_{i}}$, where $N$ is the total number of particles, $r_{i}$ is the position of the $i$ th particle, and $q$ is the wave-vector. The zero-time quantum density correlation is the quantum structure factor denoted by $S(q)$. It is a difficult task to evaluate $C_{\rho \rho}(q, t)$ directly with full quantum mechanical evolution. The ring polymer approach, which maps quantum particles to closed classical polymers, ${ }^{20}$ allows us to use the classical method of Reference-Interaction-Site-Model (RISM) to evaluate quantum correlations. For this, one defines Kubo transformed quantities. The Kubo transformed density correlation is defined as

$$
\tilde{C}_{\rho \rho}(q, t)=\frac{1}{N \beta \hbar} \int_{0}^{\beta \hbar} d \lambda\langle\hat{\rho}(-q, 0) \hat{\rho}(q, t+i \lambda)\rangle,
$$

where $\beta=\frac{1}{k_{B} T}$ is the inverse temperature, $k_{B}$ is the Boltzmann constant, and $\hbar$ is Planck's constant. The notation $\tilde{A}$ implies a
Kubo-transformed quantity, defined as $\tilde{A}(q)=\frac{1}{\beta \hbar} \int_{0}^{\beta \hbar} d \lambda e^{-\lambda \hat{H}} A(q) e^{\lambda \hat{H}}$, where $\hat{H}$ is the Hamiltonian of the system. The zero-time Kubotransformed density correlation is denoted by $\tilde{S}(q)$, known as the Kubo-transformed structure factor.

The projection operator formalism ${ }^{1,6,21}$ of Zwanzig and Mori is used to develop the QMCT to study the time-dependence of $\tilde{C}_{\rho \rho}$. The projection operator is defined as $\hat{P}=\sum_{q} \frac{|\tilde{A}(q)\rangle\langle A(-q)|}{\langle A(-q) \tilde{A}(q)\rangle}$, where $A(q)$ is the vector $\left(\hat{\rho}(q), \hat{g}_{z}(q)\right)^{T}$ and $g_{z}$ is the longitudinal component of momentum density.

Following the procedure outlined in Refs. 17 and 19, the following QMCT equation for $\tilde{C}_{\rho \rho}$ is obtained:

$$
\frac{d^{2} \tilde{C}_{\rho \rho}(q, t)}{d t^{2}}+\Omega_{q}^{2} \tilde{C}_{\rho \rho}(q, t)+\int_{0}^{t} d t^{\prime} M\left(q, t-t^{\prime}\right) \frac{d \tilde{C}_{\rho \rho}\left(q, t^{\prime}\right)}{d t^{\prime}}=0,
$$

where $\Omega_{q}^{2}=\frac{q^{2}}{m \beta \tilde{S}(q)}$ is the frequency term, $m$ is the mass of the particle, and $M(q, t)=\frac{1}{2 \pi} \int_{-\infty}^{\infty} d w e^{-i \omega t} M(q, \omega)$ with

$$
\begin{aligned}
M(q, \omega)= & \frac{\hbar m \beta^{2}}{16 \omega \pi^{3} q^{3} n} \int_{0}^{\infty} d k k \int_{|q-k|}^{q+k} d \kappa \kappa V_{q, k, \kappa}^{2} \\
& \times \int_{-\infty}^{\infty} d \omega^{\prime} \Phi_{\kappa}\left(\omega^{\prime}\right) \Phi_{k}\left(\omega-\omega^{\prime}\right) \omega^{\prime}\left(\omega-\omega^{\prime}\right) T\left(\omega^{\prime}, \omega-\omega^{\prime}\right),
\end{aligned}
$$

where $\Phi_{q}(\omega)=\int_{-\infty}^{\infty} d t e^{i \omega t} \tilde{C}_{\rho \rho}(q, t)$ is the Fourier transform of the density-density, Kubo transformed, correlation function.

The last term in Eq. (3) is the (quantum) mode-coupling nonlinear term, where $M(q, t)$ is the memory function that originates due to the correlation of random forces. The memory function is approximated by decoupling the four-point density correlation in terms of the product of two-point correlation functions, and the projected dynamics of the random force is replaced with the full dynamics projected onto the slow decaying modes. The approximated vertex function is given by ${ }^{19}$

$$
V_{q, k, \kappa}=\frac{\Delta n\left(\Omega_{\kappa}\right) \Delta n\left(\Omega_{k}\right) \zeta_{q, k, \kappa}}{S(\kappa) S(k) K\left(\Omega_{\kappa}, \Omega_{k}\right)}\left[\frac{\left(\Omega_{k}+\Omega_{\kappa}\right)^{2}-\Omega_{q}^{2}}{\Omega_{k}+\Omega_{\kappa}}\right],
$$

where

$$
\zeta_{q, k, \kappa}=\frac{\Omega_{q} S(q) S(k) S(\kappa)-\frac{\hbar \Delta n\left(\Omega_{q}\right)}{4 m}\left[\left(q^{2}+k^{2}-\kappa^{2}\right) S(\kappa)+\left(q^{2}-k^{2}+\kappa^{2}\right) S(k)\right]}{\Omega_{q} \Delta n\left(\Omega_{k}+\Omega_{\kappa}\right)-\left(\Omega_{k}+\Omega_{\kappa}\right) \Delta n\left(\Omega_{q}\right)},
$$

$$
K\left(\Omega_{\kappa}, \Omega_{k}\right)=\frac{T\left(\Omega_{\kappa}, \Omega_{k}\right)}{\Omega_{\kappa}+\Omega_{k}}+\frac{T\left(-\Omega_{\kappa}, \Omega_{k}\right)}{\Omega_{\kappa}-\Omega_{k}} .
$$

Here, $n$ is the number density, $n(w)=\frac{1}{e^{\beta \hbar w}-1}$ is the Bose distribution, $\Delta n(w)=n(w)-n(-w)$, and $T\left(w_{1}, w_{2}\right)=n\left(-w_{1}\right) n\left(-w_{2}\right)$ $-n\left(w_{1}\right) n\left(w_{2}\right)$.

In order to solve Eq. (3), we first need to evaluate the memory function in the frequency domain, which requires the pre-knowledge of the whole set of density correlations in the frequency domain, as given in Eq. (4). The numerical calculation of $M(q, \omega)$ involves the calculation of several integrals one inside another, which is computationally expensive. Once the frequencydependent memory function is obtained using some proper guess value for $\Phi_{q}(\omega)$, the memory function can be Fourier transformed to the time domain to obtain $\tilde{C}_{\rho \rho}(q, t)$. The new set of $\Phi_{q}(\omega)$ can be calculated through Fourier-transforming $\tilde{C}_{\rho \rho}(q, t)$, and the memory 
function can be updated in a self-consistent manner. These numerical calculations require back and forth frequency- and time-domain transformations, where a small error may add up in successive selfconsistent loops and lead to a wrong result or may not converge at all. These difficulties can be avoided using a perturbative approach to solve the dynamics of the quantum density correlation as we describe below.

\section{A. Perturbative approach}

Equation (4) contains a convolution of products of $\phi(q, \omega)$ with $T\left(\omega, \omega^{\prime}\right)$, which makes it difficult to analytically transform the memory term $M(q, \omega)$ in the time domain. The function $\frac{1}{\omega} \omega^{\prime}\left(\omega-\omega^{\prime}\right) T\left(\omega^{\prime}, \omega-\omega^{\prime}\right)$ present in the memory function can be expanded around $\beta \hbar$ as

$$
\begin{aligned}
& \frac{1}{\omega} \omega^{\prime}\left(\omega-\omega^{\prime}\right) T\left(\omega^{\prime}, \omega-\omega^{\prime}\right) \\
& =\frac{1}{\beta \hbar}+\frac{\beta \hbar}{12} \omega^{\prime}\left(\omega-\omega^{\prime}\right)-\frac{(\beta \hbar)^{3}}{720} \sum_{i=0}^{n=2}(-1)^{i} \omega^{\prime i+1}\left(\omega-\omega^{\prime}\right)^{n-i+1} \\
& +\frac{(\beta \hbar)^{5}}{30240} \sum_{i=0}^{n=4}(-1)^{i} \omega^{\prime i+1}\left(\omega-\omega^{\prime}\right)^{n-i+1} \\
& \quad-\frac{(\beta \hbar)^{7}}{1209600} \sum_{i=0}^{n=6}(-1)^{i} \omega^{\prime i+1}\left(\omega-\omega^{\prime}\right)^{n-i+1} \\
& +\frac{(\beta \hbar)^{9}}{47900160} \sum_{i=0}^{n=8}(-1)^{i} \omega^{\prime i+1}\left(\omega-\omega^{\prime}\right)^{n-i+1} \\
& \quad-\frac{691(\beta \hbar)^{11}}{210 \times 13 !} \sum_{i=0}^{n=10}(-1)^{i} \omega^{\prime i+1}\left(\omega-\omega^{\prime}\right)^{n-i+1}+O\left(\geq(\beta \hbar)^{13}\right)
\end{aligned}
$$

Plugging this into the memory function expression [Eq. (4)], we get

$$
\begin{aligned}
M(q, \omega)= & \frac{\hbar m \beta^{2}}{16 \pi^{3} q^{3} n} \int_{0}^{\infty} d k k \int_{|q-k|}^{q+k} d \kappa \kappa V_{q, k, \kappa}^{2} \\
& \times \int_{-\infty}^{\infty} d \omega^{\prime} \phi_{\kappa}\left(\omega^{\prime}\right) \phi_{k}\left(\omega-\omega^{\prime}\right)\left[\frac{1}{\beta \hbar}+\frac{\beta \hbar}{12} \omega^{\prime}\left(\omega-\omega^{\prime}\right)\right. \\
& \left.-\frac{(\beta \hbar)^{3}}{720} \sum_{i=0}^{n=2}(-1)^{i} \omega^{i+1}\left(\omega-\omega^{\prime}\right)^{n-i+1}+\cdots\right] .
\end{aligned}
$$

Fourier transforming Eq. (9) in the time domain, we obtain

$$
\begin{aligned}
M(q, t)= & \frac{m \beta}{8 \pi^{2} q^{3} n} \int_{0}^{\infty} d k k \int_{|q-k|}^{q+k} d \kappa \kappa V_{q, k, \kappa}^{2} \\
& \times\left[\tilde{C}_{\rho \rho}(\kappa, t) \tilde{C}_{\rho \rho}(k, t)-\frac{(\beta \hbar)^{2}}{12} \tilde{C}_{\rho \rho}^{(1)}(\kappa, t) \tilde{C}_{\rho \rho}^{(1)}(k, t)\right. \\
& \left.-\frac{(\beta \hbar)^{4}}{720} \sum_{i=0}^{n=2}(-1)^{i} \tilde{C}_{\rho \rho}^{(i+1)}(\kappa, t) \tilde{C}_{\rho \rho}^{(n-i+1)}(k, t)-\cdots\right]
\end{aligned}
$$

where $\tilde{C}_{\rho \rho}^{(i)}(q, t)$ is the $i$ th time derivative of the density-correlation function.

In the following calculations, the memory function in Eq. (10) is computed perturbatively to the 14 th power of $\beta \hbar$ and the higher power terms are ignored:

$$
\begin{aligned}
M(q, t) \approx & \frac{m \beta}{8 \pi^{2} q^{3} n} \int_{0}^{\infty} d k k \int_{|q-k|}^{q+k} d \kappa \kappa V_{q, k, \kappa}^{2} \times\left[\tilde{C}_{\rho \rho}(\kappa, t) \tilde{C}_{\rho \rho}(k, t)\right. \\
& -\frac{\Lambda^{* 2} \tau^{2}}{12} \tilde{C}_{\rho \rho}^{(1)}(\kappa, t) \tilde{C}_{\rho \rho}^{(1)}(k, t)-\frac{\Lambda^{* 4} \tau^{4}}{720} \sum_{i=0}^{n=2}(-1)^{i} \tilde{C}_{\rho \rho}^{(i+1)}(\kappa, t) \tilde{C}_{\rho \rho}^{(n-i+1)}(k, t) \\
& -\frac{\Lambda^{* 6} \tau^{6}}{30240} \sum_{i=0}^{n=4}(-1)^{i} \tilde{C}_{\rho \rho}^{(i+1)}(\kappa, t) \tilde{C}_{\rho \rho}^{(n-i+1)}(k, t)-\frac{\Lambda^{* 8} \tau^{8}}{1209600} \sum_{i=0}^{n=6}(-1)^{i} \tilde{C}_{\rho \rho}^{(i+1)}(\kappa, t) \tilde{C}_{\rho \rho}^{(n-i+1)}(k, t) \\
& -\frac{\Lambda^{* 10} \tau^{10}}{47900160} \sum_{i=0}^{n=8}(-1)^{i} \tilde{C}_{\rho \rho}^{(i+1)}(\kappa, t) \tilde{C}_{\rho \rho}^{(n-i+1)}(k, t)-\frac{691 \Lambda^{* 12} \tau^{12}}{210 \times 13 !} \sum_{i=0}^{n=10}(-1)^{i} \tilde{C}_{\rho \rho}^{(i+1)}(\kappa, t) \\
& \left.\times \tilde{C}_{\rho \rho}^{(n-i+1)}(k, t)-\frac{\Lambda^{* 14} \tau^{14}}{12 \times 13 !} \sum_{i=0}^{n=12}(-1)^{i} \tilde{C}_{\rho \rho}^{(i+1)}(\kappa, t) \tilde{C}_{\rho \rho}^{(n-i+1)}(k, t)\right]
\end{aligned}
$$

where $\Lambda^{*}=\frac{\hbar}{\sqrt{m \sigma^{2} K_{B} T}}$ is the ratio of the thermal wavelength to the particle size, $\tau=\sqrt{m \sigma^{2} \beta}, \sigma$ is the diameter, and $m$ is the mass of the particle. At the $\Lambda^{*} \rightarrow 0$ limit, the quantum vertex $V_{q, k, \kappa}$ exactly reduces to the classical one; ${ }^{9}$ thereby in this limit, quantum and classical memory functions become equal. With a gradual increase in $\Lambda^{*}$, the quantum fluctuations become important and the quantum effect is well captured by the perturbative terms up to a certain value of $\Lambda^{*}$.

In the perturbative approach, we can evaluate the memory function in the time domain and Eq. (3) can be solved in a selfconsistent manner by passing back and forth Fourier transforms to 
frequency and time domains. Having lesser number of integrals in the time-dependent memory function makes it computationally less costly compared to calculating the memory term in the frequency domain. In this method, the Kubo-transformed and quantum static structure factors are used as the input. The quantum static structure factor for the hard-sphere system is calculated using RISM, where the Percus-Yevick (PY) approximation is used as the closure equation. The Kubo-transformed structure factor is calculated using the approximate relation $\left[\tilde{S}(q) \approx \frac{2 S(q)}{\beta \hbar \Delta n\left(\Omega_{q}\right)}\right] .^{19}$

\section{B. Nonergodic parameter (NEP)}

For a liquid, the density correlation decays in time and vanishes in the long time limit. As the liquid is supercooled, the density fluctuations become correlated over much longer timescales. MCT predicts a sharp transition to the glassy state where these correlations are frozen at all times at all length scales. The system is said to be trapped in a metastable state and therefore become nonergodic. The density fluctuations at $t \rightarrow \infty$, also referred to as nonergodic parameter (NEP), identify this sharp transition. The NEP is defined as the normalized density correlation at $t \rightarrow \infty$,

$$
f_{q}=\frac{\tilde{C}_{\rho \rho}(q, t \rightarrow \infty)}{\tilde{C}_{\rho \rho}(q, t=0)}=\frac{\tilde{C}_{\rho \rho}(q, t \rightarrow \infty)}{\tilde{S}(q)},
$$

where $f_{q}=0$ for all wave-vectors indicates the ergodic state that is a liquid state, while $f_{q}>0$ indicates the non-ergodic (i.e., glass) state. obtained:

Using Eqs. (12) in (3), the following equation for NEP can be

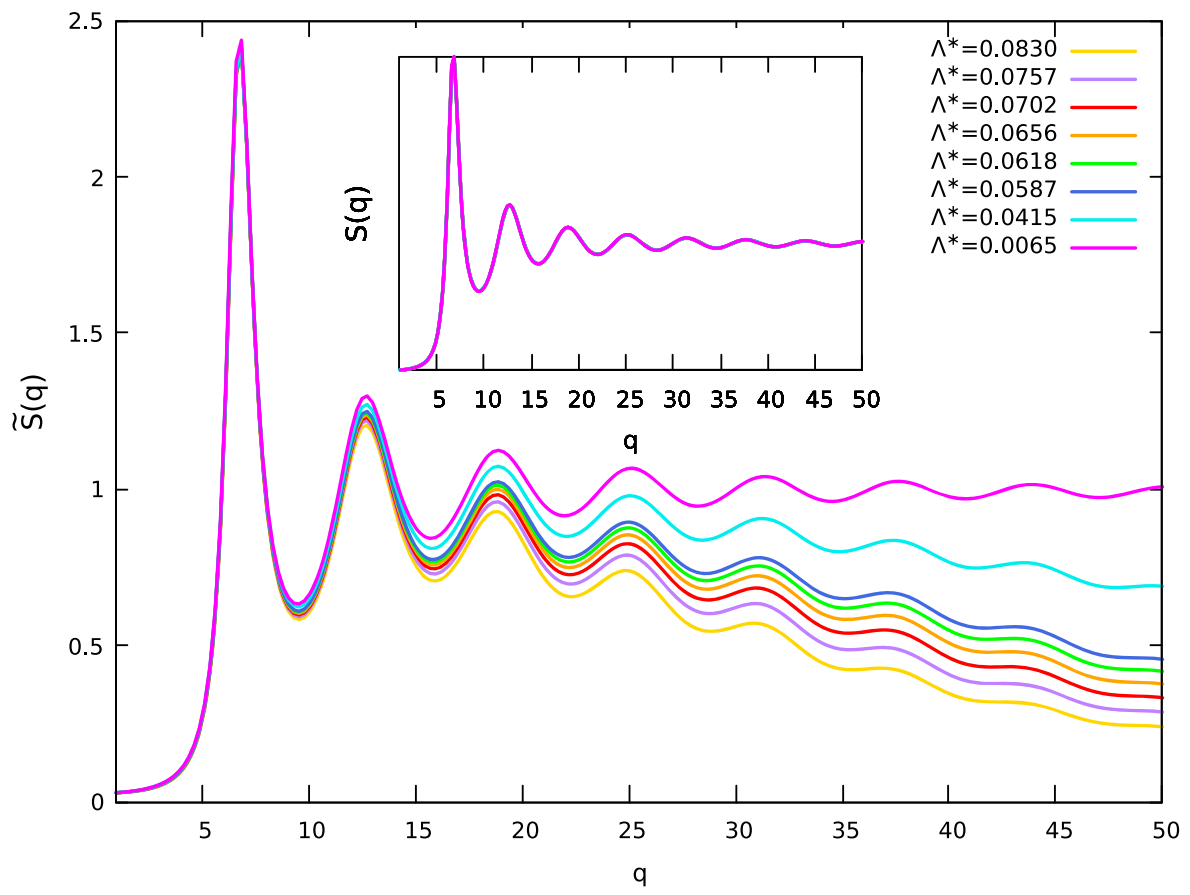

$$
f_{q}=\frac{M(q, t \rightarrow \infty)}{M(q, t \rightarrow \infty)+\Omega(q)^{2}},
$$

where $M(q, t \rightarrow \infty)$ is evaluated using Eq. (11). With $\tilde{C}_{\rho \rho}$ being constant for $t \rightarrow \infty$, the derivative terms vanish, and the long time limit of the memory function is given by

$$
M(q, t \rightarrow \infty)=\frac{m \beta}{8 \pi^{2} q^{3} n} \int_{0}^{\infty} d k k \int_{|q-k|}^{q+k} d \kappa \kappa V_{q, k, \kappa}^{2} f_{\kappa} f_{k} \tilde{S}(\kappa) \tilde{S}(k) .
$$

Equations (13) and (14) should be solved in a self-consistent manner to calculate the nonergodic parameter. This parameter determines the critical conditions for the liquid-glass transition at different degrees of quantum fluctuation. The NEP was analyzed in Ref. 18 to predict reentrant behavior of liquid-glass transition as the quantumness is varied.

\section{RESULTS}

The self-consistent approach of QMCT requires the static structure factor and Kubo transformed structure factor as the input. RISM equations with PY closure were used to generate the inputs for a single component hard sphere (HS) system. ${ }^{19}$ The degree of quantumness is measured through the dimensionless parameter $\Lambda^{*}$ defined above. By decreasing the mass or temperature of the system, we can control the value of $\Lambda^{*}$; a higher value of $\Lambda^{*}$ indicates higher quantum fluctuations in the system. Figure 1 shows the Kubo transformed structure factor for different values of $\Lambda^{*}$ at the
FIG. 1. Kubo-transformed structure factor is shown for $\eta=0.445$ for different quantum fluctuations. With the increase in $\Lambda^{*}$ values, $\tilde{S}(q)$ at a large wavevector deviates more from unity. Inset shows the quantum structure factor for different $\Lambda^{*}$ values with no visible difference for different $\Lambda^{*} . q$ is in the units of $\sigma^{-1}$. 
volume fraction $\eta=0.445$ (the classical HS system shows transition at critical $\eta_{c}=0.5089$ ). The inset shows the quantum structure factor at $\eta=0.445$ for different values of $\Lambda^{*}$. The quantum structure factor $S(q)$ is not affected much as $\Lambda^{*}$ is varied, but the Kubo-transformed structure factor $\tilde{S}(q)$ shows significant dependence on $\Lambda^{*}$, especially at larger $q$ values where it tends to decrease with the increase in $q$, which enhances as $\Lambda^{*}$ is increased. As the large value of $q$ corresponds to short length scales, the structure factor at a large $q$ gives information about the self-part of the density correlation, which, by definition, is unity for $S(q)$, as shown in the inset of the figure. The Kubo-transformed structure factor corresponds to the structure factor of a ring-polymer consisting of an infinite number of beads. ${ }^{20}$ Upon increasing the quantumness, the polymer becomes more flexible, leading to more uncertainty in the position of the quantum particle, which leads to smaller values for the Kubo correlation as $q$ is increased.

Using $S(q)$ and $\tilde{S}(q)$ as inputs, we solve Eqs. (13) and (14) selfconsistently to obtain NEPs and the critical density for different $\Lambda^{*}$ values. Some results for NEP are shown in Fig. 2 at $\eta=0.445$ for various values of $\Lambda^{*}$. In the inset, we reproduce the results of Ref. 18 for the reentrant behavior of liquid-glass transition as $\Lambda^{*}$ is varied. The NEP in blue corresponds to $\Lambda^{*}=0.1016$, which is the liquid-glass transition point for $\eta=0.445$ marked with blue arrow in the inset. If $\Lambda^{*}$ is increased along the line of $\eta=0.445$, the first peak of $f_{q}$ rises up to $\Lambda^{*}=0.415$, and on further increasing $\Lambda^{*}$, the peak height of $f_{q}$ starts decreasing. Beyond $\Lambda^{*}=0.757$, the $f_{q}$ is zero for all $q$ values and the system reenters the liquid phase. Increasing $\Lambda^{*}$ further, the transition point shifts to lower values of density, and beyond $\Lambda^{*} \approx 0.3$, the critical density starts to move toward the classical value. We find that beyond $\Lambda^{*}>1$, the transition occurs at densities higher than the classical value. For $\Lambda^{*}=2.39$, the liquid-glass transition occurs at $\eta_{c}=0.623$, which is considerably high compared to classical critical density and close to the value $\eta$ $=0.74$ for a closed pack system (e.g., face centered cubic lattice). We realize that at such high densities, the current results may not be very reliable as the PY-approximation used to generate the static structure may fail. However, due to quantum effects, it should be possible to go to higher densities retaining the supercooled liquid phase. The phase diagram suggests the existence of two different kinds of liquids: one at the region of lower $\Lambda^{*}$ dominated by classical interaction and the other in the region of higher $\Lambda^{*}$ dominated by the enhanced quantum tunneling effect. We are able to calculate the dynamics of the supercooled liquid of the first kind of liquid in a low $\Lambda^{*}$ region $\left(\Lambda^{*} \leq 0.092\right)$ along the red curve at $\eta=0.445$ in the inset. This limitation is due to the use of perturbative calculation as described above. It will be interesting to explore the dynamic and structural differences in the liquids in the two extreme values of quantumness. This will require going beyond the perturbative approach and will be discussed elsewhere.

In Fig. 3, the normalized Kubo density correlation is plotted against time, $t$ (in units of $\tau$ ), for $q=7.11$ (wave-vector around the first peak of the structure factor) and $\eta=0.445$. The black dotted curve represents the dynamics of the classical hard-sphere system, and the solid curves represent the dynamics of the quantum supercooled liquid. Results for $\Lambda^{*}=0.0065$ match with the classical dynamics and represent the classical limit of the quantum system. Like classical density correlation, the Kubo correlation also shows two-step relaxation: $\beta$-relaxation (the plateau region) governed by power-law decay, followed by $\alpha$-relaxation (tail region) dictated by stretched exponential decay. As the quantum fluctuation increases, the time taken for the correlation to decay gradually increases. The increase in $\Lambda^{*}$ in the lower region of quantumness

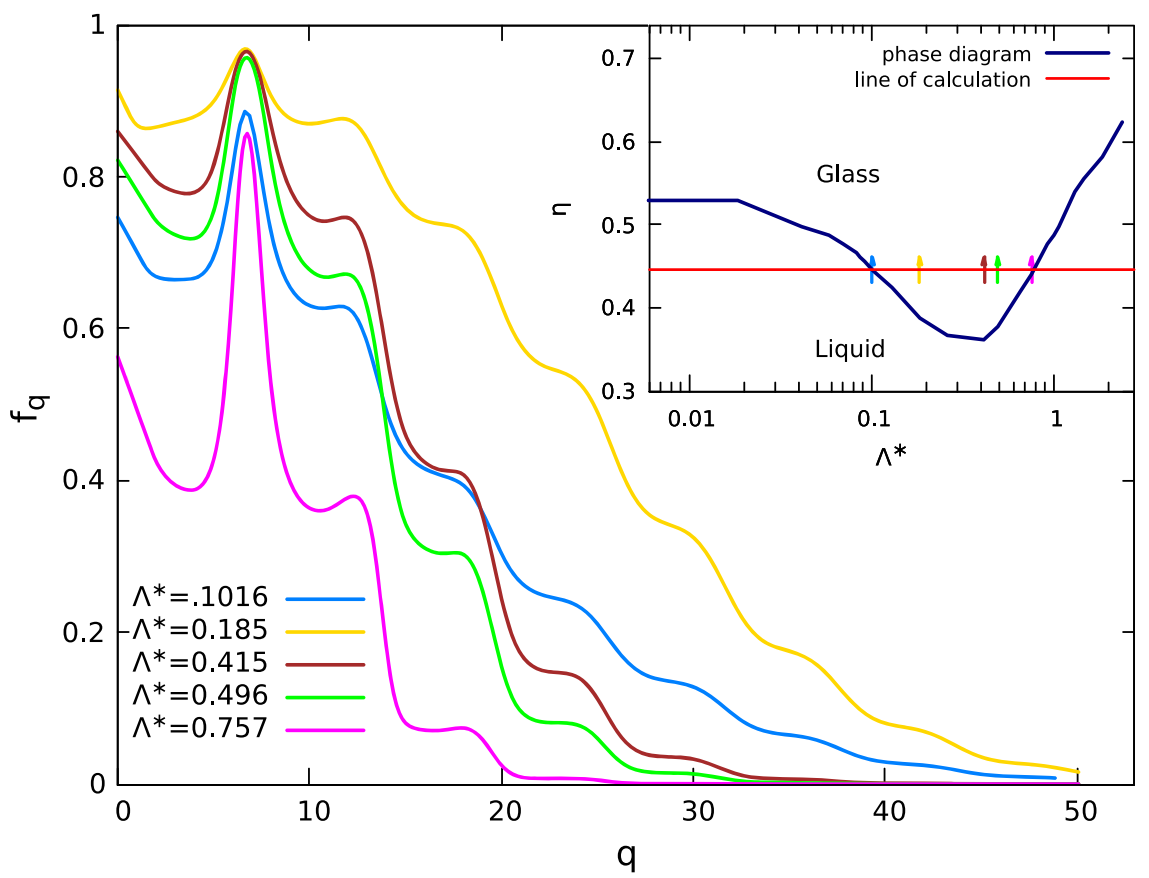

FIG. 2. Non-ergodicity parameter at $\eta$ $=0.445$ as a function of $q$ is plotted for a varying degree of quantum fluctuation. Inset shows the phase diagram for liquid-glass transition with increasing quantum fluctuation. The red line is at $\eta=0.445$, along which the dynamics is calculated. The positions of the NEPs in $\Lambda^{*}$ are shown on the red line with small arrows. 


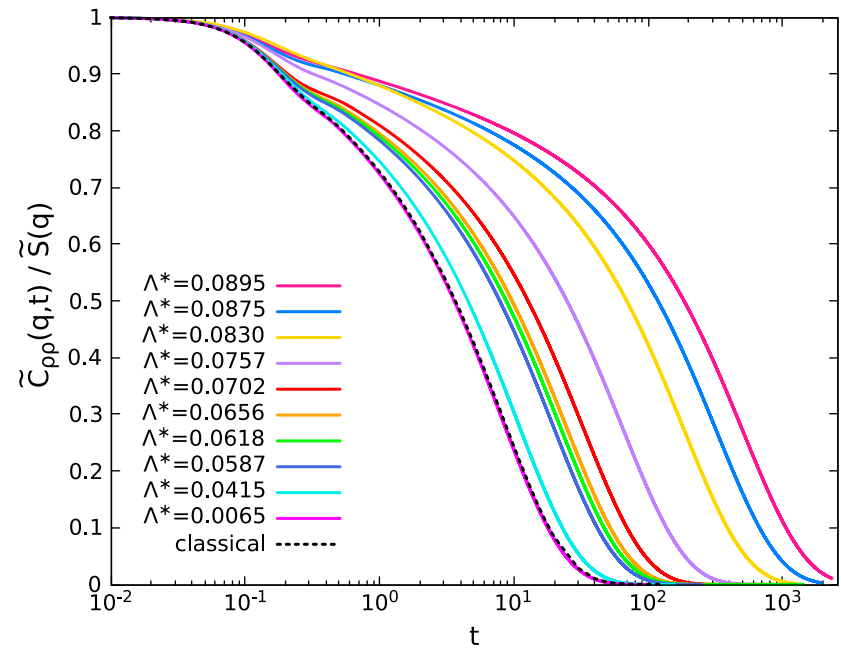

FIG. 3. Dynamics of the density correlation is plotted at volume fraction $\eta=0.445$ and $q=7.11$ with different degrees of quantum fluctuation. The black dotted curve represents the classical dynamics. Dynamics slows down with the increase in quantum fluctuation

indicates increased dimensions of the ring-polymer, and thereby, the particles (each ring-polymer) experience more cage effect (jammed state) by their neighboring particles resulting in slower relaxation.

In Fig. 4, we show divergence in the relaxation time, $t_{\alpha}$, as the critical value of $\eta$ is approached. The relaxation time is approximated with the time where the correlation function decays to $1 / e \approx 0.367879$ of its initial value. The QMCT data for the relaxation time is fitted with power-law $t_{\alpha}=t_{0}\left(\frac{1}{\eta_{c}-\eta}\right)^{v}$, with $t_{0}$ and $v$ being the fit parameters. The critical values of $\eta_{c}$ are obtained from NEP calculation from QMCT. In Fig. 4(a), we present results for the increase in the relaxation time as the density is increased, keeping $\Lambda^{*}$ fixed. The blue and the black dots correspond to $\Lambda^{*}=0.0587$ and 0.0830 , respectively, for which the critical densities are $\eta_{c}=0.483$ and 0.466 , respectively. The two power-law fits give different exponents. For $\Lambda^{*}$ $=0.0587, v=2.81$, while for $\Lambda^{*}=0.0830, v=2.96$. It is known that for the classical HS ${ }^{22}$ and Lennard-Jones (LJ) liquids, ${ }^{23,24}$ the relaxation time increases near the transition point following power-law behavior with $v=2.46$ and $v \sim 2.5$, respectively, as the (HS) density is increased or the (LJ) temperature is decreased. For comparison, we also show results for the classical $\left(\Lambda^{*}=0\right)$ case [shown with open circles in Fig. 4(a)] for which $v=2.46$. The above results indicate that, similar to the classical liquid, the relaxation time in quantum liquid also follows power-law divergence quite well as the density is increased, although the power-law exponent, $v$, varies with the quantumness. To explore it further, we compute the power-law exponent for different values of $\Lambda^{*}$. The exponent increases almost linearly as $\Lambda^{*}$ increases from zero. This is depicted in Fig. 4(b).

We next discuss divergence in the relaxation time, $t_{\alpha}$, as critical value of $\Lambda^{*}$ is approached at fixed density. The variation in the relaxation time with $\Lambda^{*}$ is shown in Fig. 5. The data are fitted with the power law $t_{\alpha}=t_{0}\left(\frac{1}{\Lambda_{c}^{*}-\Lambda^{*}}\right)^{v}$. The critical values of $\Lambda_{c}^{*}$ are obtained from the NEP calculation from QMCT. For $\eta=0.445$ and 0.4608 , $\Lambda_{c}^{*}=0.1016$ and 0.08675 , respectively. The black and the blue circles show QMCT data, and the lines show power-law fits. We find that for $\eta=0.445$, the power-law exponent $v=2.59$, whereas for a higher density, $\eta=0.4608$, the power-law exponent decreases to 2.41 .

Note that in the moderate quantum regime, the critical value of $\Lambda^{*}$ is higher for lower densities (see the inset in Fig. 2). Thus in Fig. 5, the data for the smaller density correspond to the higher quantumness compared to the data for the higher density. Since the power-law exponent is greater for larger $\Lambda^{*}$, a faster divergence (larger power-law exponent) is seen for the smaller density.
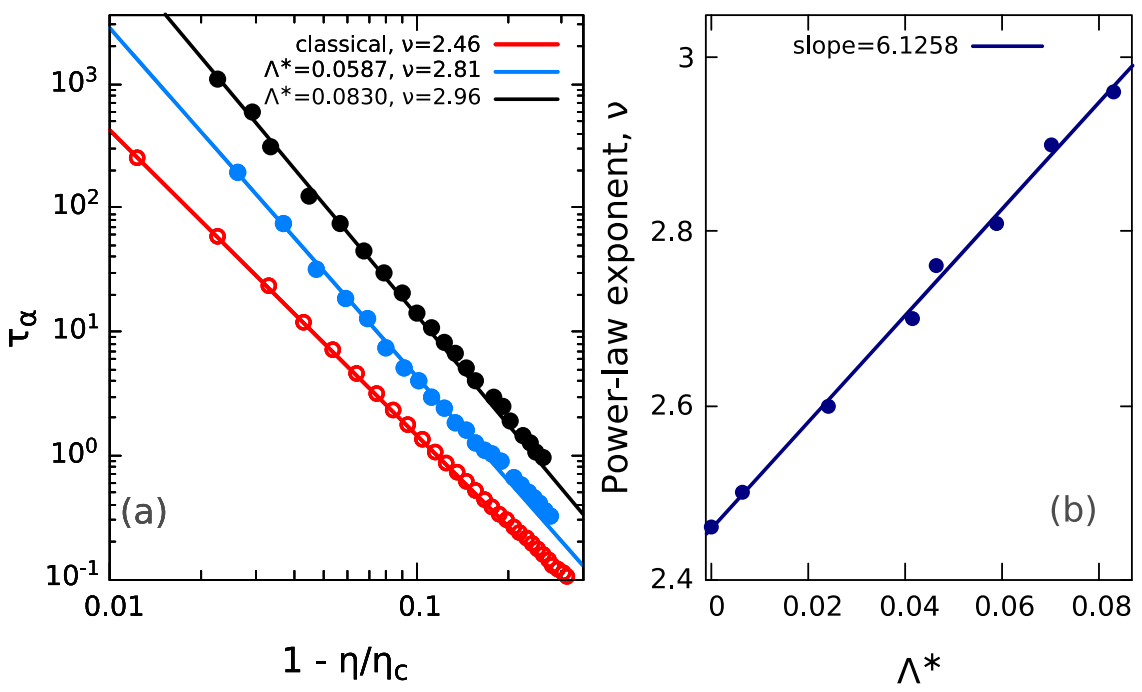

FIG. 4. (a) Increase in the relaxation time, $t_{\alpha}$, as $\eta$ is increased at fixed $\Lambda^{*}$. $\eta_{c}$ is the critical value obtained from QMCT. Dots represent the QMCT results, the solid black and the blue curves show power-law fits, and the red curve with void circles is the classical result. The red and the blue data points are shifted vertically for clarity by an amount $-\ln (7)$ and $-\ln (2.4)$, respectively. (b) Variation in the power-law exponent $v$ as the quantumness is increased. 


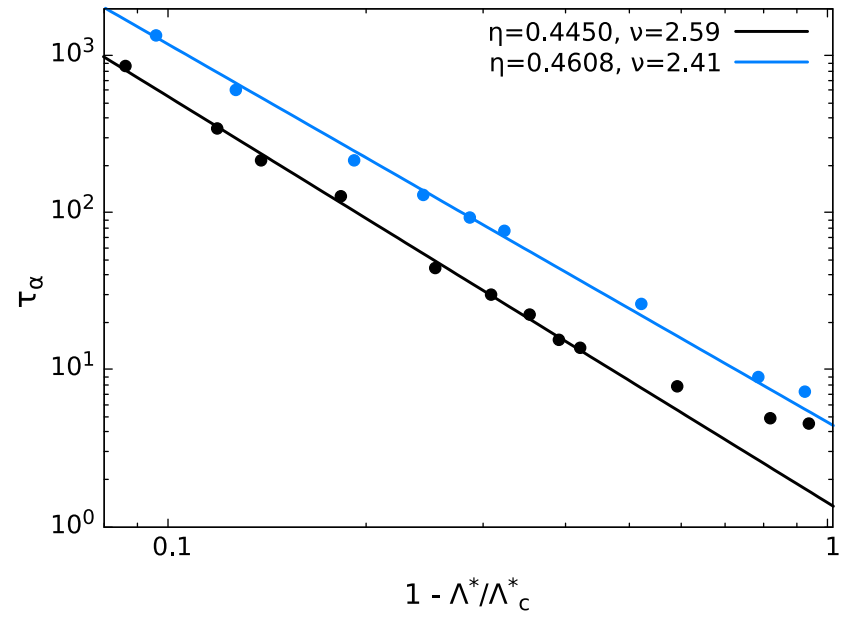

FIG. 5. Increase in the relaxation time with the increase in $\Lambda^{*}$ at fixed densities $\Lambda_{c}^{*}$ is the critical value obtained from QMCT. Dots represent the QMCT results and the black and blue solid curves show power-law fit.

For the quantum case $\left(\Lambda^{*}>0\right)$, the power law fits well close to the transition in Fig. 5 but deviates over smaller values of the quantumness. We find that a Vogel-Fulcher-Tammann (VFT)-like form, $t_{\alpha}=t_{0} \exp \left[\frac{A}{\left(\Lambda_{c}^{*}-\Lambda^{*}\right)^{\delta}}\right]$, can be used to describe increase in the relaxation time quite well over the entire $\Lambda^{*}$ range considered. This is shown in Fig. 6 for the same data points, as reported in Fig. 5. For $\eta=0.445$ and 0.4608 , the VFT exponents are $\delta=0.300$ and 0.297 , respectively. The VFT, however, does not describe the increase in relaxation time with respect to the density $x=\eta_{c}-\eta$. For comparison, we fit VFT to the data of Fig. 4(a). The fit is shown in the inset of Fig. 6. It is clear that the VFT fit is not as good as the power-law fit shown in Fig. 4(a).

\section{CONCLUSION}

We have used quantum mode-coupling theory to study dynamics in supercooled hard sphere quantum liquids. The quantumness of the system can be tuned to manipulate the glass-transition point in the system. For relatively high quantumness $\left(\Lambda^{*}>1\right)$, the critical density can surpass the critical density for classical liquid and may reach close to the closed-pack density. This is because the enhanced quantum effects lead to pronounced tunneling to overcome the classical caging effect and allow the system to remain in the ergodic phase up to higher densities. In the moderate quantum regime $\left(\Lambda^{*}\right.$ $\leq 0.1$ ), the relaxation time, for fixed $\Lambda^{*}$, increases with density and shows power-law divergence near the critical point. This divergence becomes stronger upon increasing the quantumness $\left(\Lambda^{*}\right)$.

The dynamical analysis presented in this work is based on a perturbative treatment of the quantum vertex function, which is valid in the moderate quantum regime. For the long time dynamics, the derivative terms in Eq. (11) become less important. At $t \rightarrow \infty$, all derivative terms are strictly zero, and the memory function simplifies and takes the form of the memory function obtained in the classical MCT. However, in QMCT, the vertex function within the memory function contains quantum effects through $\Lambda^{*}$. Although the absolute value of the density correlation function is sensitive to the derivative terms, the power-law, which is expected to emerge close to the transition point and is a characteristic of long-time relaxation, is not affected significantly by the derivative terms, which become insignificant at long times.

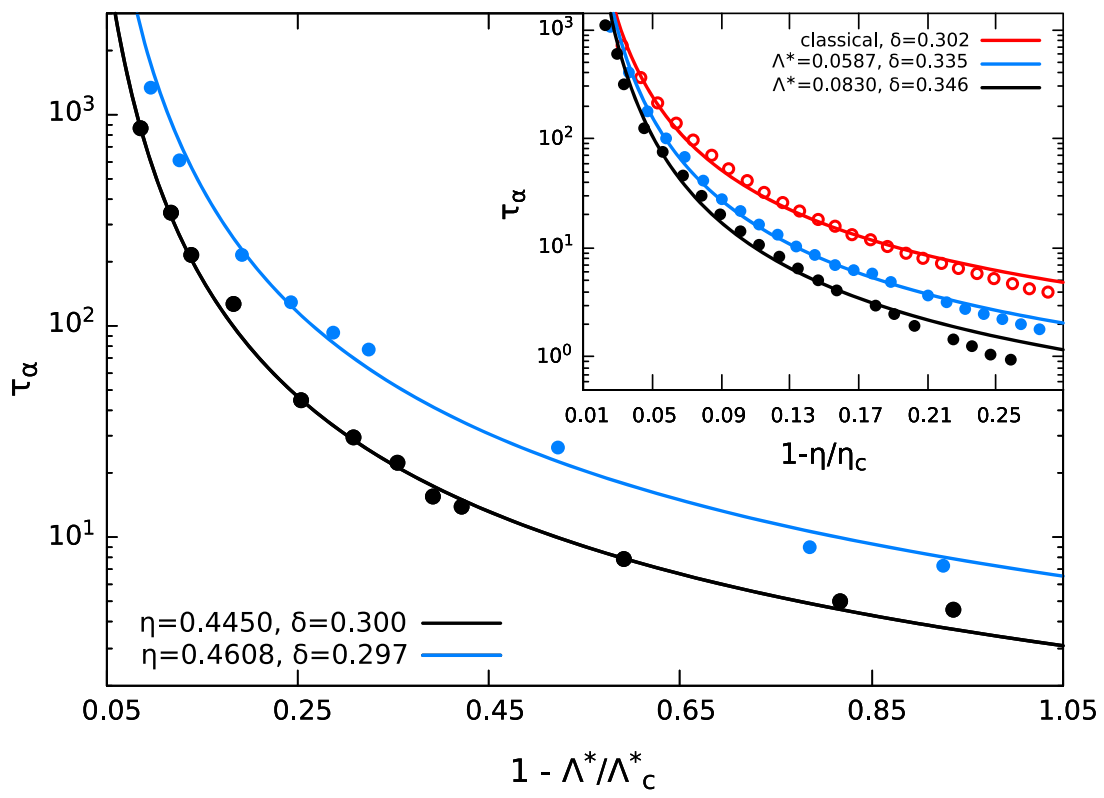

FIG. 6. Vogel-Fulcher-Tammann (VFT)like fit $t_{\alpha}=t_{0} \exp \left[\frac{A}{\left(\Lambda_{c}^{*}-\Lambda^{*}\right)^{\delta}}\right]$ to the relaxation time, $t_{\alpha}$, as $\Lambda^{*}$ is increased at fixed $\eta$. Inset shows the VFT fit to the relaxation time as $\eta$ is varied for fixed $\Lambda^{*}$. The red and the blue data points have been shifted vertically for clarity by $\ln (4.3)$ and $\ln (2.3)$, respectively. 


\section{ACKNOWLEDGMENTS}

A.D. acknowledges the support from the University Grants Commission (UGC), India. U.H. acknowledges the support from IISc under TATA Trust Travel Fund. K.M. acknowledges the support by the Japan Society for the Promotion of Science (JSPS) KAKENHI (Grant Nos. 16H04034 and 20H00128).

\section{DATA AVAILABILITY}

The data that support the findings of this study are available from the corresponding author upon reasonable request.

\section{REFERENCES}

${ }^{1}$ J.-P. Hansen and I. R. McDonald, Theory of Simple Liquids, 4th ed. (Academic Press, Oxford, 2013)

${ }^{2}$ C. A. Angell, J. Non-Cryst. Solids 131-133, 13 (1991).

${ }^{3}$ B. Kim and Gene F. Mazenko, Adv. Chem. Phys. 78, 129 (1990).

${ }^{4}$ W. Gotze and L. Sjogren, Rep. Prog. Phys. 55, 241 (1992).

${ }^{5}$ S. P. Das, "The supercooled liquid," in Statistical Physics of Liquids at Freezing and Beyond (Cambridge University Press, 2011), pp. 164-203.

${ }^{6}$ D. R. Reichman and P. Charbonneau, J. Stat. Mech.: Theory Exp. 2005, P05013.
${ }^{7}$ W. Götze, Condens. Matter Phys. 1, 873 (1998)

${ }^{8}$ S. P. Das and G. F. Mazenko, Phys. Rev. A 34, 2265 (1986).

${ }^{9}$ U. Bengtzelius, W. Gotze, and A. Sjolander, J. Phys. C: Solid State Phys. 17, 5915 (1984).

${ }^{10}$ U. Harbola and S. P. Das, Phys. Rev. E 65, 036138 (2002)

${ }^{11}$ J. S. Thakur and J. Bosse, Phys. Rev. A 43, 4378 (1991).

${ }^{12}$ U. Harbola and S. P. Das, Phys. Rev. E 64, 046122 (2001)

${ }^{13}$ R. C. Zeller and R. O. Pohl, Phys. Rev. B 4, 2029 (1971).

${ }^{14}$ J.-F. Berret, M. Meissner, and B. Mertz, Z. Phys. B 87, 213 (1992).

${ }^{15}$ S. Franz, T. Maimbourg, G. Parisi, and A. Scardicchio, Proc. Natl. Acad. Sci. U. S. A. 116, 13768 (2019).

${ }^{16}$ W. Götze and M. Lücke, Phys. Rev. B 13, 3825 (1976).

${ }^{17}$ E. Rabani and D. R. Reichman, J. Chem. Phys. 116, 6271 (2002).

${ }^{18}$ T. E. Markland, J. A. Morrone, B. J. Berne, K. Miyazaki, E. Rabani, and D. R. Reichman, Nat. Phys. 7, 134 (2011).

${ }^{19}$ T. E. Markland, J. A. Morrone, K. Miyazaki, B. J. Berne, D. R. Reichman, and E. Rabani, J. Chem. Phys. 136, 074511 (2012).

${ }^{20}$ D. Chandler and P. G. Wolynes, J. Chem. Phys. 74, 4078 (1981).

${ }^{21}$ R. Zwanzig, Nonequilibrium Statistical Mechanics (Oxford University Press, New York, 2001).

${ }^{22}$ T. Franosch, M. Fuchs, W. Götze, M. R. Mayr, and A. P. Singh, Phys. Rev. E 55, 7153 (1997).

${ }^{23}$ W. Kob and H. C. Andersen, Phys. Rev. Lett. 73, 1376 (1994).

${ }^{24}$ W. Kob and H. C. Andersen, Phys. Rev. E 52, 4134 (1995). 